\title{
Lattice gauge theory model for graphene
}

\author{
Alessandro Giuliani, ${ }^{1}$ Vieri Mastropietro, ${ }^{2}$ and Marcello Porta ${ }^{3}$ \\ ${ }^{1}$ Università di Roma Tre, L.go S. L. Murialdo 1, 00146 Roma, Italy \\ ${ }^{2}$ Università di Roma Tor Vergata, Viale della Ricerca Scientifica, 00133 Roma, Italy \\ ${ }^{3}$ Università di Roma La Sapienza, P.le A. Moro 2, 00185 Roma, Italy
}

(Received 31 August 2010; published 27 September 2010)

\begin{abstract}
The effects of the electromagnetic (em) electron-electron interactions in half-filled graphene are investigated in terms of a lattice gauge theory model. By using exact renormalization group methods and lattice Ward identities, we show that the em interactions amplify the responses to the excitonic pairings associated to a Kekulé distortion and to a charge-density wave. The effect of the electronic repulsion on the Peierls-Kekulé instability, usually neglected, is evaluated by deriving an exact non BCS gap equation, from which we find evidence that strong em interactions among electrons facilitate the spontaneous distortion of the lattice and the opening of a gap.
\end{abstract}

DOI: 10.1103/PhysRevB.82.121418

PACS number(s): 71.10.Hf, 71.30.+h, 73.63.Bd, 64.60.ae

Graphene, a monocrystalline graphitic film that has been recently experimentally realized, ${ }^{1}$ has highly unusual electronic properties, due to the quasirelativistic nature of its charge carriers. ${ }^{2}$ In the presence of long-ranged interactions, graphene provides an ideal laboratory for simulating quantum field theory (QFT) models at low energies and to possibly observe phenomena such as spontaneous chiral symmetry breaking and mass generation. For a few years, there was essentially no experimental signature of electron-electron interactions in graphene, due to substrate-induced perturbations that obscured their effects. However, the realization of suspended graphene samples is allowing people to collect increasing evidence of interaction effects. ${ }^{3}$ On the theoretical side, understanding the properties of a system of interacting fermions on the honeycomb lattice is a challenging problem, similar to quantum electrodynamics but with some peculiar differences that make its study new and nontrivial; ${ }^{4}$ its comprehension is essential for graphene and, at the same time, it has relevance for other planar condensed-matter systems, like high $T_{c}$ superconductors, and even for basic questions in QFT.

Most theoretical analyses deal with a simplified effective continuum model of massless Dirac fermions with static Coulomb interactions. Early results based on lowest-order perturbation theory predicted a growth of the effective Fermi velocity $v(\mathbf{k})$ close to the Fermi points and excluded the spontaneous formation of a gap at weak coupling. ${ }^{5(a)}$ The absence of a gap is a serious drawback for possible technological applications of graphene. Therefore, people started to investigate possible mechanisms for its generation. One way to induce a gap is by adding an interaction with an external periodic field, which can be generated, e.g., by the presence of a substrate. ${ }^{6}$ In the absence of a substrate, Ref. 7 proposed a mechanism similar to the one at the basis of spontaneous chiral symmetry breaking in strongly coupled $\mathrm{QED}_{3}$; the applicability of these proposals to real graphene is a delicate issue, due to the uncontrolled approximations related to a large-N expansions. Another possible mechanism for gap generation is based on a Peierls-Kekule distortion of the honeycomb lattice, which is a prerequisite for electron fractionalization. ${ }^{8,9}$ One optimizes over the distortion pattern, by minimizing the corresponding electronic energy. In the absence of interactions, a rather strong interaction with the classical phonon field is needed for the formation of a nontrivial distortion ${ }^{8}$ while the effects of the electron interactions are still poorly understood.

In this Rapid Communication, we investigate the effects of electronic interactions in terms of a lattice gauge theory model. We show that the electronic interactions amplify the response functions associated to Kekule (K) or charge density wave (CDW) pairings. Moreover, we derive the exact form of the Peierls-Kekule gap equation in the presence of the electromagnetic (em) electron-electron interactions, from which we find evidence that strong em interactions enhance the Peierls-Kekule instability (despite the growth of the Fermi velocity, ${ }^{10}$ which apparently opposes this effect). The model is analyzed by the methods of constructive QFT, which have already proved effective in obtaining rigorous nonperturbative results in many similar problems; ${ }^{11}$ we rely neither on the effective Dirac description nor on a large-N expansion.

The charge carries in graphene are described by tightbinding electrons on a honeycomb lattice coupled to a threedimensional (3D) quantum em field. We introduce creation and annihilation fermionic operators $\psi_{\vec{x}, \sigma}^{ \pm}=\left(a_{\vec{x}, \sigma}^{ \pm}, b_{\vec{x}+\vec{\delta}_{1}, \sigma}^{ \pm}\right)$ $=|\mathcal{B}|^{-1} \int_{\vec{k} \in \mathcal{B}} d \vec{k} \psi_{\vec{k}, \sigma}^{ \pm} e^{ \pm i \vec{k} \vec{x}}$ for electrons with spin index $\sigma=\uparrow \downarrow$ sitting at the sites of the two triangular sublattices $\Lambda_{A}$ and $\Lambda_{B}$ of a honeycomb lattice; we assume that $\Lambda_{A}=\Lambda$ has basis vectors $\vec{l}_{1,2}=\frac{1}{2}(3, \pm \sqrt{3})$ and that $\Lambda_{B}=\Lambda_{A}+\vec{\delta}_{j}$ with $\vec{\delta}_{1}=(1,0)$ and $\vec{\delta}_{2,3}=\frac{1}{2}(-1, \pm \sqrt{3})$ the nearest-neighbor vectors; $\mathcal{B}$ is the first Brillouin zone. The honeycomb lattice is embedded in $\mathrm{R}^{3}$ and belongs to the plane $x_{3}=0$. The grand-canonical Hamiltonian at half filling is $H=H_{0}+H_{C}+H_{A}$, where

$$
H_{0}=-t \sum_{\vec{x} \in \Lambda_{A}} \sum_{j, \sigma} a_{\vec{x}, \sigma}^{+} b_{\vec{x}+\vec{\delta}_{j}, \sigma}^{-} e^{i e \int_{0}^{1} \vec{\delta}_{j} \cdot \vec{A}\left(\vec{x}+s \vec{\delta}_{j}, 0\right) d s}+\text { c.c. }
$$

with $t$ the hopping parameter and $e$ the electric charge; the coupling with the em field is obtained via the Peierls substitution. Moreover, if $n_{\vec{x}}=\Sigma_{\sigma} a_{\vec{x}, \sigma}^{+} a_{\vec{x}, \sigma}^{-}$(respectively, $n_{\vec{x}}$ $=\Sigma_{\sigma} b_{\vec{x}, \sigma}^{+} b_{\vec{x}, \sigma}^{-}$) for $\vec{x} \in \Lambda_{A}$ (respectively, $\vec{x} \in \Lambda_{B}$ ), 


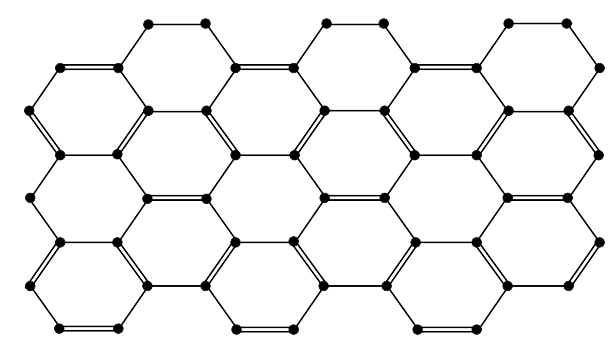

FIG. 1. The Kekule pattern; the hopping parameter is $t+\Delta_{0}$ and $t-\Delta_{0} / 2$ on the double and single bonds, respectively.

$$
H_{C}=\frac{e^{2}}{2} \sum_{\vec{x}, \vec{y} \in \Lambda_{A} \cup \Lambda_{B}}\left(n_{\vec{x}}-1\right) \varphi(\vec{x}-\vec{y})\left(n_{\vec{y}}-1\right),
$$

where $\hat{\varphi}_{\vec{p}}$ is an ultraviolet regularized version of the static Coulomb potential. Finally, $H_{A}$ is the energy (in the presence of an ultraviolet cutoff) of the 3D photon field $\underline{A}=\left(\vec{A}, A^{3}\right)$ in the Coulomb gauge. We fix units so that the speed of light $c=1$ and the free Fermi velocity $v=\frac{3}{2} t \ll 1$. If we allow distortions of the honeycomb lattice, the hopping becomes a function of the bond length $\ell_{\vec{x}, j}$ that, for small deformations, can be approximated by the linear function $t_{\vec{x}, j}=t+\phi_{\vec{x}, j}$ with $\phi_{\vec{x}, j}=g\left(\ell_{\vec{x}, j}-\bar{\ell}\right)$ and $\bar{\ell}$ the equilibrium length of the bonds. The Kekulé dimerization pattern corresponds to, see Fig. 1,

$$
\phi_{\vec{x}, j}=\phi_{0}+\Delta_{0} \cos \left[\vec{p}_{F}^{+}\left(\vec{\delta}_{j}-\vec{\delta}_{j_{0}}-\vec{x}\right)\right]
$$

with $j_{0} \in\{1,2,3\}$. In order to investigate the effect of the em interactions and the Peierls-Kekulé instability, we use the following strategy: we first compute the ground-state energy and the correlations for $\phi_{\vec{x}, j}=0$ by exact renormalization group (RG) methods, ${ }^{12}$ finding, in agreement with previous analyses, ${ }^{5(\mathrm{~b})}$ that the quasiparticle weight vanishes at the Fermi points and the effective Fermi velocity tends to the speed of light as power laws with nonuniversal critical exponents. In addition, the analysis of the response functions and of the corresponding exponents indicates a tendency toward excitonic pairing; the mass terms of $\mathrm{K}$ or CDW type are strongly amplified by the interactions. Next, we compute the electronic correlations in the presence of a nontrivial lattice distortion $\phi_{\vec{x}, j}$ and we show that a Kekulé dimerization pattern Eq. (2) is a stationary point of the total energy (i.e., the sum of the elastic energy and the electronic energy in the Born-Oppenheimer approximation).

We start with $\phi_{\vec{x}, j}=0$; the analysis is very similar to the one performed in the continuum Dirac approximation in Ref. 13 (which we refer to for more details), the main difference being that the present lattice gauge theory model is automatically ultraviolet finite and gauge invariant: this avoids the need for an ultraviolet regularization, which can lead to wellknown ambiguities. ${ }^{14}$ The $n$-points imaginary-time correlations can be obtained by the generating functional

$$
e^{W(J, \lambda)}=\int P(d \psi) \int P(d A) e^{\mathcal{V}(A+J, \psi)+(\psi, \lambda)},
$$

where: $\psi_{\mathbf{k}}^{ \pm}$are Grassman variables, with $\mathbf{k}=\left(k_{0}, \vec{k}\right)$ and $k_{0}$ the Matsubara frequency, and $P(d \psi)$ is the fermionic Gaussian integration with inverse propagator

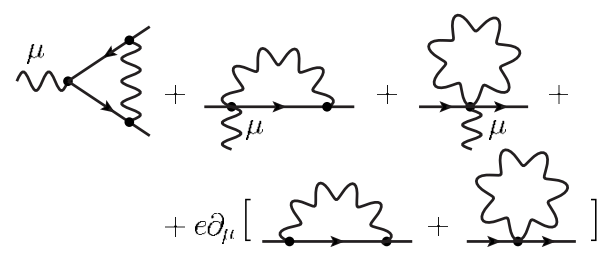

FIG. 2. The second-order graphs contributing to the dressed charge $e_{-\infty}$, given by the contribution of the vertex part minus the graphs coming from the wave-function renormalization (if $\mu=0$ ) or the velocity renormalization $(\mu=1,2)$; their sum is exactly vanishing, in agreement with the WI Eq. (5).

$$
g^{-1}(\mathbf{k})=-\frac{1}{Z}\left(\begin{array}{cc}
i k_{0} & v \Omega^{*}(\vec{k}) \\
v \Omega(\vec{k}) & i k_{0}
\end{array}\right)
$$

with $Z=1$ and $\Omega(\vec{k})=\frac{2}{3} \sum_{j=1,2,3} e^{i \vec{k}\left(\vec{\delta}_{j}-\vec{\delta}_{1}\right)}$ [note that $g(\mathbf{k})$ is singular only at the Fermi points $\left.\mathbf{k}=\mathbf{k}_{F}^{ \pm}=\left(0, \frac{2 \pi}{3}, \pm \frac{2 \pi}{3 \sqrt{3}}\right)\right]$; if $\mu$ $=0,1,2, A^{\mu}(\mathbf{p})$ are Gaussian variables with propagator $w_{\mu \nu}(\mathbf{p})=\delta_{\mu \nu} \int \frac{d p_{3}}{(2 \pi)} \frac{\chi\left(|\vec{p}|^{2}+p_{3}^{2}\right)}{\mathbf{p}^{2}+p_{3}^{2}}$, where $\chi$ is an ultraviolet cutoff function; finally $\mathcal{V}=e Z \int\left[j_{0} A_{0}+v \vec{j} \vec{A}\right]+$ h.o.t., where h.o.t. indicates higher order interaction terms in $A$ produced by the Taylor expansion of the exponential in $H_{0}$ and $j_{\mu}$ is the bare lattice current. ${ }^{15}$ We compute $W(J, \lambda)$ via a rigorous Wilsonian $\mathrm{RG}$ scheme, writing the fields $\psi, A$ as sums of fields $\psi^{(k)}, A^{(k)}$, living on momentum scales $\left|\mathbf{k}-\mathbf{k}_{F}^{ \pm}\right|,|\mathbf{p}| \simeq M^{k}$ with $k \leq 0$ a scale label and $M>1$ a scaling parameter; the iterative integration of the fields on scales $h<k \leq 0$ leads to an effective theory similar to Eq. (3) with an ultraviolet cutoff around the Fermi points of width $M^{h}$ and with effective scale-dependent wave-function renormalization $Z_{h}$, Fermi velocity $v_{h}$, and effective charge $e_{h}$. This approach works only if $e_{h}$ does not flow to strong coupling; the boundedness of $e_{h}$ follows from an exact Ward identity (WI), derived by the lattice phase transformation $\psi_{\mathbf{x}, \sigma}^{ \pm} \rightarrow e^{ \pm i e \alpha_{\mathbf{x}}} \psi_{\mathbf{x}, \sigma}^{ \pm}$in $W_{h}(0, \lambda)^{16}$

$$
\mathbf{p}_{\mu} \Lambda_{\mu}^{(h)}(\mathbf{k}, \mathbf{p})=e\left[S_{\mathbf{k}+\mathbf{p}}^{-1} \Gamma_{0}(\vec{k}, \vec{p})-\Gamma_{0}(\vec{k}, \vec{p}) S_{\mathbf{k}}^{-1}\right],
$$

where $S_{\mathbf{k}}$ is the interacting propagator, $\Gamma_{0}(\vec{k}, \vec{p})=\left(\begin{array}{cc}-i & 0 \\ 0 & -i e^{-i \vec{p} \delta_{1}}\end{array}\right)$ and $\Lambda_{\mu}^{(h)}(\mathbf{k}, \mathbf{p})$ is the vertex function ${ }^{17}$ that, if computed at external momenta $\left|\mathbf{k}-\mathbf{k}_{F}^{ \pm}\right| \sim M^{h}$ and $|\mathbf{p}| \ll M^{h}$, is proportional to $e_{h}$ (if $\mu=0$ ) or $e_{h} v_{h}$ (if $\mu=1,2$ ). Using Eq. (5), we find that $e_{h} \rightarrow e_{-\infty}=e+e^{3} F(e)$ with $F(e)$ a series in $e^{2}$ with bounded coefficients, i.e., the effective charge tends to a line of fixed points; $F(e)$ is vanishing at lowest order, see Fig. 2, but the WI does not exclude that $F(e)$ is nonzero at higher orders. A similar WI implies that the photon remain massless and, as an outcome of the above procedure, we get an expansion of the Schwinger functions in the effective couplings $e_{h}$ that is finite at all orders, see Ref. 13 for the proof; this is in contrast with the naive perturbation theory, which is plagued by logarithmic divergencies. The boundedness of $e_{h}$ makes such expansion meaningful and it allows one to control the flow of $Z_{h}$ and $v_{h}$. One finds that: (i) $\lim _{h \rightarrow-\infty} v_{h}=1$, i.e., Lorentz invariance spontaneously emerges; (ii) both $Z_{h}^{-1}$ and $1-v_{h}$ vanish with two anomalous power laws, see Ref. 13. Therefore, if $\phi_{\vec{x}, j}=0$, the dressed propagator has a form similar to 
Eq. (4) with $Z$ and $v$ replaced by $Z\left(\mathbf{k}-\mathbf{k}_{F}^{ \pm}\right)$and $v\left(\mathbf{k}-\mathbf{k}_{F}^{ \pm}\right)$; if $\mathbf{k}$ is far from the Fermi points, $Z\left(\mathbf{k}-\mathbf{k}_{F}^{ \pm}\right)$and $v\left(\mathbf{k}-\mathbf{k}_{F}^{ \pm}\right)$are close to their unperturbed values, namely, 1 and $\frac{3}{2} t$. On the contrary, if $\left|\mathbf{k}^{\prime}\right| \ll 1, Z\left(\mathbf{k}^{\prime}\right) \sim\left|\mathbf{k}^{\prime}\right|^{-\eta}$ with $\eta=\frac{e^{2}}{12 \pi^{2}}+\cdots$ an anomalous critical exponent that is finite at all orders in $e^{2}$, and $v\left(\mathbf{k}^{\prime}\right)$ tends to the speed of light. Moreover, $1-v\left(\mathbf{k}^{\prime}\right)$ $\sim(1-v)\left|\mathbf{k}^{\prime}\right|^{\tilde{\eta}}$ with $\tilde{\eta}=\frac{2 e^{2}}{5 \pi^{2}}+\cdots$ another anomalous critical exponent.

The above analysis confirms, at all orders and in the presence of a lattice cutoff, the results found long ago in Ref. 5(b), where graphene was described by an effective continuum Dirac model with an ultraviolet dimensional regularization; the exponents agree at lowest order. Gauge invariance implies that the dressed Fermi velocity has a universal value (the speed of light) and Lorentz invariance emerges; this is what happens both in Ref. 5(b) (thanks to the use of dimensional regularization) and in the present more realistic lattice model, thanks to the exact lattice WI Eq. (5). On the other hand, we expect that as soon as gauge invariance is broken the limiting Fermi velocity is smaller than the speed of light; this is indeed what happens in Ref. 13, where gauge invariance is broken by the momentum cutoff.

In order to understand which instabilities are likely to occur in the system, we compute response functions or generalized susceptibilities (which apparently have never been systematically computed before not even in the Dirac approximation); more precisely, for $\phi_{\vec{x}, j}=0$, we compute $R^{(\alpha)}\left(\mathbf{x}, j ; \mathbf{y}, j^{\prime}\right)=\left\langle\rho_{\mathbf{x}, j}^{(\alpha)} \rho_{\mathbf{y}, j^{\prime}}^{(\alpha)}\right\rangle \quad$ with, $\quad$ e.g., $\quad \rho_{\mathbf{x}, j}^{(K)}$ $=\Sigma_{\sigma}\left(a_{\mathbf{x}, \sigma}^{+} b_{\mathbf{x}+\left(0, \vec{\delta}_{j}\right), \sigma}^{-} e^{i e \int_{0}^{1} \vec{\delta}_{j} \cdot \vec{A}\left(\mathbf{x}+s\left(0, \vec{\delta}_{j}\right), 0\right) d s}+\right.$ c.c. $) \quad$ or $\quad \rho_{\mathbf{x}, j}^{(\mathrm{CDW})}$ $=\Sigma_{\sigma}\left(a_{\mathbf{x}, \sigma}^{+} a_{\mathbf{x}, \sigma}^{-}-b_{\mathbf{x}+\left(0, \vec{\delta}_{j}\right), \sigma}^{+} b_{\mathbf{x}+\left(0, \vec{\delta}_{j}\right), \sigma}^{-}\right)$. The two latter operators describe, respectively, internode and intranode excitonic pairings of $\mathrm{K}$ and $\mathrm{CDW}$ type; this is because the possible presence of a condensate in the $\mathbf{k}=\mathbf{k}_{F}^{ \pm}$channel for $\rho_{\mathbf{x}, j}^{(K)}$ or in the $\mathbf{k}=\mathbf{0}$ channel for $\rho_{\mathbf{x}, j}^{(\mathrm{CDW})}$ would signal the emergence of long range order (LRO) of K-type (see Fig. 1) or of CDW type (a period-2 alternation of excess/deficit of electrons in the sites of the A/B lattice). Other relevant bilinears are the Cooper pairings, i.e., linear combinations of terms of the form $a_{\mathbf{x}, \sigma}^{+} a_{\mathbf{x},-\sigma}^{+}$or $a_{\mathbf{x}, \sigma}^{+} b_{\mathbf{x}+\left(0, \vec{\delta}_{j}\right), \sigma^{\prime}}^{+}$. The large distances asymptotic behavior of the response functions is

$$
R^{(\alpha)}(\mathbf{x}, j ; \mathbf{0}, j) \sim G_{1}^{(\alpha)}(\mathbf{x})+\cos \left(\vec{p}_{F}^{+} \cdot \vec{x}\right) G_{2}^{(\alpha)}(\mathbf{x})
$$

with $\left|G_{i}^{(\alpha)}(\mathbf{x})\right| \sim($ const $)|\mathbf{x}|^{-\xi_{i}^{(\alpha)}}$ two scaling invariant functions (similar formulas are valid for $j \neq j^{\prime}$ ). In the absence of interactions, $\xi_{i}^{(\alpha)}=4$, for all $\alpha$ and $i$; there are no preferred instabilities. The presence of the interaction with the em field removes the degeneracy in the decay exponents: some responses are enhanced and some other depressed. It turns out that

$$
\xi_{1}^{(\mathrm{CDW})}=4-4 e^{2} /\left(3 \pi^{2}\right)+\cdots, \quad \xi_{2}^{(\mathrm{K})}=4-4 e^{2} /\left(3 \pi^{2}\right)+\cdots
$$

On the contrary, $\xi_{2}^{(\mathrm{CDW})}$ and $\xi_{1}^{(\mathrm{K})}$ are vanishing at second order while all the Cooper pairs responses decay faster than $|\mathbf{x}|^{-4}$. The conclusion is that the responses to excitonic pairing of $\mathrm{K}$ or CDW type are amplified by the em interaction: in this sense, we can say that the em interaction induces quasi LRO of $\mathrm{K}$ and CDW type.

Correspondingly, possible small distortions or inhomogeneities of the K or CDW type are dramatically enhanced by the interactions. For instance, let us choose $\phi_{\vec{x}, j}$ as in Eq. (2). The RG analysis can be repeated in the presence of a Kekulé mass term $\Delta_{0} \Sigma_{\vec{x}, j} \cos \left[p_{F}^{+} \cdot\left(\vec{x}-\vec{\delta}_{j}+\vec{\delta}_{j_{0}}\right)\right] \rho_{\vec{x}, j}^{(\mathrm{K})}$ in the Hamiltonian, which produces a new relevant coupling constant, the effective Kekulé mass. Therefore, the interaction produces an effective momentum-dependent gap $\Delta(\mathbf{k})$ that increases with a power law with exponent $\eta^{\mathrm{K}}$ from the value $\Delta_{0}$ up to

$$
\Delta\left(\mathbf{k}_{F}^{ \pm}\right)=\Delta_{0}^{1 /\left(1+\eta^{\mathrm{K}}\right)}, \quad \eta^{\mathrm{K}}=2 e^{2} /\left(3 \pi^{2}\right)+\cdots .
$$

Note that the ratio of the dressed and bare gaps diverges as $\Delta_{0} \rightarrow 0$. The enhancement of the dressed gap is related to the phenomenon of gap generation in Ref. 7 but it is found here avoiding any unrealistic large- $N$ expansion. A similar enhancement is found for the gap due to a CDW modulation.

Finally, let us discuss a possible mechanism for the spontaneous distortion of the lattice and the opening of a gap (Peierls-Kekulé instability). We use a variational argument, which shows that a Kekulé dimerization pattern of the form Eq. (2) is a stationary point of the total energy $\frac{\kappa}{2 g^{2}} \sum_{\vec{x}, j} \phi_{\vec{x}, j}^{2}$ $+E_{0}\left(\left\{\phi_{\vec{x}, j}\right\}\right)$, where the first term is the elastic energy and $E_{0}\left(\left\{\phi_{\vec{x}, j}\right\}\right.$ is the electronic ground-state energy in the BornOppenheimer approximation. The extremality condition for the energy is $\kappa \phi_{\vec{x}, j}=g^{2}\left\langle\rho_{\vec{x}, j}^{(\mathrm{K})}\right\rangle^{\phi}$, where $\langle\cdot\rangle^{\phi}$ is the ground-state average in the presence of the distortion pattern $\left\{\phi_{\vec{x}, j}\right\}$. Computing $\left\langle\rho_{\vec{x}, j}^{(\mathrm{K})}\right\rangle^{\phi}$ by RG with the multiscale analysis explained above, we find that Eq. (2) is a stationary point of the total energy, provided that $\phi_{0}=c_{0} g^{2} / \kappa+\cdots$ for a suitable constant $c_{0}$ and that $\Delta_{0}$ satisfies the following non BCS gap equation:

$$
\Delta_{0} \simeq \frac{g^{2}}{\kappa} \int_{\Delta \leqslant\left|\mathbf{k}^{\prime}\right| \lesssim 1} d \mathbf{k}^{\prime} \frac{Z^{-1}\left(\mathbf{k}^{\prime}\right) \Delta\left(\mathbf{k}^{\prime}\right)\left|\Omega\left(\vec{k}^{\prime}\right)\right|^{2}}{k_{0}^{2}+v^{2}\left(\mathbf{k}^{\prime}\right)\left|\Omega\left(\vec{k}^{\prime}+\vec{p}_{F}^{\omega}\right)\right|^{2}+\left|\Delta\left(\mathbf{k}^{\prime}\right)\right|^{2}},
$$

where $\Delta=\Delta_{0}^{1 /\left(1+\eta^{\mathrm{K}}\right)}$ and, for $\Delta \lesssim\left|\mathbf{k}^{\prime}\right| \ll 1, Z\left(\mathbf{k}^{\prime}\right) \sim\left|\mathbf{k}^{\prime}\right|^{-\eta}, 1$ $-v\left(\mathbf{k}^{\prime}\right) \sim(1-v)\left|\mathbf{k}^{\prime}\right|^{\tilde{\eta}}$, and $\Delta\left(\mathbf{k}^{\prime}\right) \simeq \Delta_{0}\left|\mathbf{k}^{\prime}\right|^{-\eta^{K}}$. In the absence of interactions, $Z\left(\mathbf{k}^{\prime}\right)=1, v\left(\mathbf{k}^{\prime}\right)=v$, and the above equation reduces to the free one in Ref. 8. Our gap equation is qualitatively equivalent to the simpler expression

$$
1=g^{2} \int_{\Delta}^{1} d \rho \frac{\rho^{\eta-\eta_{\mathrm{K}}}}{1-(1-v) \rho^{\tilde{\eta}}}
$$

from which its main features can be easily inferred.

At weak em coupling, the integral in the right-hand side (r.h.s.) is infrared convergent, which implies that a nontrivial solution is found only for $g$ larger than a critical coupling $g_{c}$; remarkably, $g_{c} \sim \sqrt{v}$ with $v$ the free Fermi velocity, even though the effective Fermi velocity flows to the speed of light. Therefore, at weak coupling, the prediction for $g_{c}$ is qualitatively the same as in the free case ${ }^{8}$ this can be easily checked by noting that the denominator in the r.h.s. of Eq. (7) is sensibly different from $v$ only if $\rho$ is exponentially small in $v / \tilde{\eta}$.

On the other hand, if one trusts our gap equation also at 
strong em coupling and if in such a regime $\eta^{\mathrm{K}}-\eta=\frac{7 e^{2}}{12 \pi^{2}}$ $+\cdots$ exceeds one, then the r.h.s. of Eq. (7) diverges as $\Delta$ $\rightarrow 0$, which guarantees the existence of a nontrivial solution for arbitrarily small $g$; this can be easily checked by rewriting Eq. (7), up to smaller corrections, as $1 \simeq \frac{g^{2}}{\eta^{\mathrm{K}}-\eta-1} \Delta^{1+\eta-\eta^{\mathrm{K}}}$, that is $\Delta_{0} \simeq g^{2\left(1+\eta^{\mathrm{K}}\right) /\left(\eta^{\mathrm{K}}-\eta-1\right)}$ : note the non BCS form of the gap, similar to the one appearing in certain Luttinger superconductors. ${ }^{18}$ The existence of a nontrivial solution for arbitrarily small $g$ suggests that strong em interactions between fermions enforce the Peierls-Kekulé mechanism and facilitate the spontaneous distortion of the lattice and the gap generation, by lowering the critical phonon coupling $g_{c}$; this is in agreement with the one-dimensional case, where the Peierls instability is enhanced by the electronic repulsion, see Ref. 19. Note the crucial role in the above discussion played by the momentum dependence of the gap term [leading to the factor $\rho^{\eta-\eta_{\mathrm{K}}}$ in Eq. (7)]; on the contrary, the growth of the
Fermi velocity plays a minor role. A similar analysis can be repeated for the gap generated by a CDW instability.

In conclusion, we considered a lattice gauge theory model for graphene and we predicted that the electron repulsion enhances dramatically, with a nonuniversal power law, the gaps due to the Kekulé distortion or to a density asymmetry between the two sublattices, as well as the responses to the corresponding excitonic pairings. Moreover, we derived an exact non BCS gap equation for the Peierls-Kekulé instability from which we find evidence that strong em interactions facilitate the spontaneous distortion of the lattice and the gap generation, by lowering the critical phonon coupling.

A.G. and V.M. gratefully acknowledge financial support from the ERC under Grant No. CoMBoS-239694. We thank D. Haldane and M. Vozmediano for many valuable discussions.
${ }^{1}$ K. S. Novoselov, A. K. Geim, S. V. Morozov, D. Jiang, Y. Zhang, S. V. Dubonos, I. V. Grigorieva, and A. A. Firsov, Science 306, 666 (2004); K. S. Novoselov, A. K. Geim, S. V. Morozov, D. Jiang, M. I. Katsnelson, I. V. Grigorieva, S. V. Dubonos, and A. A. Firsov, Nature (London) 438, 197 (2005); Y. Zhang, Y. W. Tan, H. L. Stormer, and P. Kim, ibid. 438, 201 (2005).

${ }^{2}$ A. H. Castro Neto, F. Guinea, N. M. R. Peres, K. S. Novoselov, and A. K. Geim, Rev. Mod. Phys. 81, 109 (2009).

${ }^{3}$ X. Du, I. Skachko, F. Duerr, A. Luican, and E. Y. Andrei, Nature (London) 462, 192 (2009); K. I. Bolotin, F. Ghahari, M. D. Shulman, H. L. Stormer, and P. Kim, ibid. 462, 196 (2009); Z. Q. Li, E. A. Henriksen, Z. Jiang, Z. Hao, M. C. Martin, P. Kim, H. L. Stormer, and D. N. Basov, Nat. Phys. 4, 532 (2008).

${ }^{4}$ G. W. Semenoff, Phys. Rev. Lett. 53, 2449 (1984); F. D. M. Haldane, ibid. 61, 2015 (1988).

5 (a) J. González, F. Guinea, and M. A. H. Vozmediano, Phys. Rev. B 59, R2474 (1999); (b) Nucl. Phys. B 424, 595 (1994).

${ }^{6}$ S. Y. Zhou, G.-H. Gweon, A. V. Fedorov, P. N. First, W. A. de Heer, D.-H. Lee, F. Guinea, A. H. Castro Neto, and A. Lanzara, Nature Mater. 6, 770 (2007).

${ }^{7}$ D. V. Khveshchenko and H. Leal, Nucl. Phys. B 687, 323 (2004); E. V. Gorbar, V. P. Gusynin, V. A. Miransky, and I. A. Shovkovy, Phys. Rev. B 66, 045108 (2002); I. F. Herbut, Phys. Rev. Lett. 97, 146401 (2006).
${ }^{8}$ C.-Y. Hou, C. Chamon, and C. Mudry, Phys. Rev. Lett. 98, 186809 (2007).

${ }^{9}$ R. Jackiw and S.-Y. Pi, Phys. Rev. Lett. 98, 266402 (2007).

${ }^{10}$ D. T. Son, Phys. Rev. B 75, 235423 (2007).

${ }^{11}$ A. Giuliani and V. Mastropietro, Commun. Math. Phys. 293, 301 (2010); Phys. Rev. B 79, 201403(R) (2009).

${ }^{12}$ G. Benfatto and G. Gallavotti, J. Stat. Phys. 59, 541 (1990).

${ }^{13}$ A. Giuliani, V. Mastropietro, and M. Porta, Ann. Henri Poincaré (to be published).

${ }^{14}$ E. G. Mishchenko, EPL 83, 17005 (2008); I. Herbut, V. Juricic, and O. Vafek, Phys. Rev. Lett. 100, 046403 (2008); I. Herbut, V. Juricic, O. Vafek, and M. Case, arXiv:0809.0725 (unpublished).

${ }^{15} j_{\mu}=\int \frac{d \mathbf{k}}{2 \pi|\mathcal{B}|} \frac{d \mathbf{p}}{(2 \pi)^{3}} \psi_{\mathbf{k}+\mathbf{p}}^{+} \Gamma_{\mu}(\mathbf{k}, \mathbf{p}) \psi_{\mathbf{k}}^{-}$where $\Gamma_{\mu}$ are matrices which can be deduced from Eq. (1) with $\Gamma_{0}\left(\mathbf{k}_{F}^{\omega}, 0\right)=-i I, \Gamma_{1}\left(\mathbf{k}_{F}^{\omega}, 0\right)=-\sigma_{2}$, $\Gamma_{2}\left(\mathbf{k}_{F}^{\omega}, 0\right)=-\omega \sigma_{1}$.

${ }^{16} W_{h}$ is defined in the same way as $W$ with an extra infrared cutoff suppressing momenta smaller than $M^{h}$.

${ }^{17}$ More precisely, $\quad \mathbf{p}_{\mu}\left[S_{\mathbf{k}+\mathbf{p}} \Lambda_{\mu}^{(h)}(\mathbf{k}, \mathbf{p}) S_{\mathbf{k}}\right]_{i j}=\frac{\partial}{\partial \alpha_{\mathbf{p}}} \frac{\partial^{2}}{\partial \lambda_{\mathbf{k}, j} \partial \lambda_{\mathbf{k} \mathbf{p}, i}^{+}}$ $\left.W_{h}(\partial \alpha, \lambda)\right|_{\alpha=\lambda=0}$.

${ }^{18}$ V. Mastropietro, Mod. Phys. Lett. B 13, 585 (1999); E. W. Carlson, D. Orgad, S. A. Kivelson, and V. J. Emery, Phys. Rev. B 62, 3422 (2000).

${ }^{19}$ E. B. Kolomeisky and J. P. Straley, Phys. Rev. B 53, 12553 (1996). 